\title{
Search for Heavy Majorana Neutrinos in Events with Same-Sign Lepton Pairs and Jets Using the CMS Detector in pp Collisions at $\sqrt{s}=13 \mathrm{TeV}$
}

\author{
Jae Sung Kim ${ }^{* \dagger}$ \\ Seoul National University (KR) \\ E-mail: jae.sung.kimecern.ch
}

\begin{abstract}
We present searches on Heavy Majorana neutrinos in events with same-sign lepton pairs and jets, using the collision data collected with the CMS detector at a centre-of-mass energy $13 \mathrm{TeV}$. The search range is extended to $20 \mathrm{GeV}$ and $1500 \mathrm{GeV}$, lower and upper bound respectively, compared to the previous analysis using $8 \mathrm{TeV}$ data. The vector boson fusion production channel is considered as well as the s-channel, which improves the sensitivities for mass above by several hundreds of GeV. We set upper limits on muon mixing squared, electron mixing squared, and muon-electron cross mixing.
\end{abstract}

The 39th International Conference on High Energy Physics (ICHEP2018)

4-11 July, 2018

Seoul, Korea

* Speaker.

${ }^{\dagger}$ CMS Collaboration 


\section{Introduction}

The discovery of neutrino oscillations [1] is a clear evidence of physics beyond the standard model. The Type-I seesaw mechanism is one of the simplest models which explains both the origin and the smallness of the mass of neutrinos. We performed a new search for type-I seesaw heavy Majorana neutrinos in the ee, $\mu \mu$, and e $\mu$ channels using CMS [2] data collected in 2016 at $\sqrt{s}=13 \mathrm{TeV}$.

\section{Event Selection}

We used dilepton triggers, and collected events which contain two same-sign leptons with jets. We further defined two signal regions which are more sensitive to low- and high-mass signals. Additional optimized selections are applied for each signal mass hypothesis.

\section{Backgrounds}

Prompt same-sign lepton backgrounds coming from multibosons, pair-produced top quarks associated with vector bosons, and double-parton scattering are estimated from simulation. Fake leptons generated by hadron decays, photon conversions, or punch through to the muon systems are estimated with a data-driven method. Finally, the mismeasured-sign background is also measured with a data-driven method, and is found to be negligible except for the ee channel.

\section{Result and Interpretations}
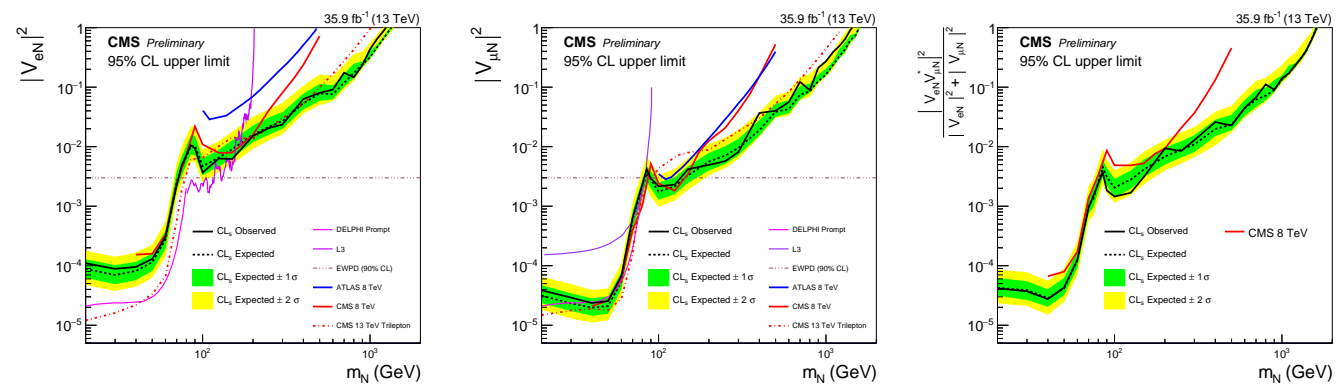

Figure 1: Exclusion limits at 95\% confidence level for dielectron, dimuon and electron-muon channels [3].

Exclusion limits at 95\% confidence level are set on the heavy Majorana neutrino mixing matrix elements as a function of the mass of the neutrino. Results for each channel are shown in Fig. 1.

\section{References}

[1] Super-Kamiokande Collaboration, Evidence for Oscillation of Atmospheric Neutrinos, Phys. Rev. Lett., 81 (1998) 1562.

[2] CMS Collaboration, The CMS experiment at the CERN LHC, JINST 3:S08004, 2008.

[3] CMS Collaboration, Search for heavy Majorana neutrinos in the same-sign dilepton channel in proton-proton collisions at $\sqrt{s}=13 \mathrm{TeV}, \mathrm{CMS}-\mathrm{EXO}-17-028$, [hep-ex/1806.10905]. 